\title{
THz absorption spectra of glucose and its polymers
}

\author{
$\underline{\text { Anna Semenova }}^{1}$, Yu.S. Guseva ${ }^{1,2}$ V.L. Vaks ${ }^{1,2}$, A.N. Panin ${ }^{1}$, D.A. Babarina ${ }^{2}$, S.S. Morunova ${ }^{3}$, \\ A.S. Vilkov ${ }^{3}$ \\ ${ }^{1}$ Institute for Physics of Microstructures, Nizhny Novgorod, Russia, semenanna@yahoo.com \\ ${ }^{2}$ Lobachevsky State University, Nizhny Novgorod, Russia, \\ ${ }^{3}$ Nizhny Novgorod State Agricultural Academy, Nizhny Novgorod, Russia
}

\section{Introduction}

Terahertz (THz) spectroscopy has tremendous potential for biological and medical applications. First, THz spectroscopy is used for chemical analysis of multicomponent gas mixture, particularly, for exhaled breath (EB) analysis. There are narrow absorption lines in the $\mathrm{THz}$ range caused by quantum transitions between rotation levels of molecules in gas phase and different lines are corresponded with different chemical substances. Because the exhaled breath is a mixture of not only oxygen, nitrogen and carbon dioxide but more than 800 gases which concentrations reflect the level of homeostasis, as well as the severity of pathological conditions, EB analysis is a perspective method for diagnostic purposes. It is non-invasive and unpainful for patients. The probability of infection transmission is very low for the method because there is no contact with any biological fluids. It is rather inexpensive and very sensitive for small concentration of measured substances. Therefore, $\mathrm{THz}$ spectroscopy of exhaled breath is becoming a powerful method of medical diagnostics, especially, incipient stage diagnostics and prevention of socially important diseases $[1,2]$.

Second, THz technic could provide a powerful tool for investigating and even manipulating of biomolecules in situ, in water solution, and in vivo, in cell cultures and whole organisms. $\mathrm{THz}$ irradiation is no ionizing and is treated as non invasive for biomolecules [2, 3] if irradiation power and exposure times do not exceed some threshold. Worth noting that absorption of organic samples and liquid water in the $\mathrm{THz}$ frequency range is caused by low-frequency vibration modes of the molecules, which occur than large groups of atoms of the molecule move coherent, and hydrogen bond net oscillation in the sample. Hydrogen bonds stabilize spatial structure of biomolecules whereas which determine eigen frequencies of the molecular vibration modes. Therefore, $\mathrm{THz}$ spectra are unique for each spatial structure of each molecule [2 - 6]. Worth noting that biomolecular spatial structures commonly described in the terms of configuration and conformation are crucial for their functions. Configuration means the mutual bracing of chemical bonds in a molecule, it can not change without chemical reaction. For example, $\mathrm{R}$ and $\mathrm{S}$ forms of any chiral molecule differs as left and right hand corresponds to different conformations. It is well known that even $\mathrm{R}$ and $\mathrm{S}$ isomers of rather simple molecules such as amino acids represent different pharmacological effects [7]. Conformation means momental spatial displacement of atoms in a molecule, it continuously oscillating near some equilibrium state by thermal fluctuations without any chemical reactions. Conformational state and dynamics determine biomolecular activity. For example, specificity of enzyme catalysis is caused by matching of space structure and electric charge displacement between the enzyme and substrate molecules; structural changes occurring at denaturation lead to loss of catalytic activity (notable that denaturation could be accompanied by no chemical conversion) [7, wiki 8]. Some conformational transitions are lethally dangerous, for example, PrP protein abnormal forms, so-called prions, stop execute their common functions and begin catalyze only PrP molecules conformational transmission from normal to the same abnomal form causing neurodegenerative diseases [прионы, 9]. Therefore there is necessary medicine task developing conformational diagnostics of biomolecules in situ, for example in water solutions, and in vivo, for example, in yeast cells for understanding how exactly they manage to control their specific prions [elements 10]. However, commonly used at present methods such as X-ray analysis, electronic and atomic force microscopy requires molecular crystals or adsorbed molecules. On the one hand, $\mathrm{THz}$ spectroscopy is becoming the urgent method investigating of conformational state and dynamics of biomolecules in liquid media that minimizes the influence on the explored molecules [2 - 6] On the other hand, there are announcements about non-thermal influence of strong $\mathrm{THz}$ field on cell life activity, cellular differentiation and animals behaviour [4, 5]. The reported effects manifested frequency dependance so powerful and frequency stable $\mathrm{THz}$ sources could be used for exciting specific conformational transitions, for example, for conversion from prion to normal form of PrP protein [11, 12].

\section{Purposes}

While THz spectroscopy shows great perspectives for diagnostics of molecular spatial structure, 
nevertheless its practical application is obstructed by some problems mainly caused by rather short story of $\mathrm{THz}$ technic and, consequently, rather small experience in the field. There are not enough models pointing at what to look at in $\mathrm{THz}$ absorption spectra. Our work is aimed at comparative analysis of absorption spectra measured for water solutions of different carbohydrates with different concentrations. The explored samples include water solutions of monosaccharides glucose and fructose, disaccharide sucrose and polysaccharide amylose.

First investigation of dependance absorption spectra on concentration is planned for the glucose solutions with concentration varying from $10 \%$ to $40 \%$. There is some possibility of deviation from the most common linear dependance because $\mathrm{THz}$ absorption of water-water and water-glucose hydrogen bonds differs and dependance of the amount of both types of bonds on the solution concentration is not linear at high concentrations.

Second investigation of molecular configuration influence on absorption spectra is planned for two isomers, glucose and fructose, prepared in form of water solutions with the same concentration.

Third there is planned absorption spectra comparison for water solutions of glucose and its polymer amylose for finding out any specific corresponds to structure of long linear molecule.

Final there is planned to compare the spectrum of solution containing $20 \%$ glucose and $20 \%$ fructose with the spectrum of $40 \%$ sucrose solution. A molecule of fructose could be hydrolyzed into two molecules (one glucose and one fructose) but in neutral solutions at room temperature both glucose-fructose mixture and sucrose are stable. Thus difference between the two spectra could be caused by difference between molecular structures with resembling chemical composition.

\section{Experimental setup}

The principal scheme of the experimental setup is shown on fig. 1, where $\mathrm{S}$ means source of monochromatic $\mathrm{THz}$ irradiation with frequency turnable in the range from 112,5 to $114,8 \mathrm{GHz}, \mathrm{D}$ means detector of $\mathrm{THz}$ irradiation and $\mathrm{C}$ means cell with liquid sample or pure water (control).

The distance between the cell and the detector was fixed while the distance between the cell and the source was variated. The detected power depends quasi periodically on the distance due to standing wave forming. There are two measurements for the each frequency and each sample with two different distances between the source and the cell corresponded to two neighbour maximums of detected power.

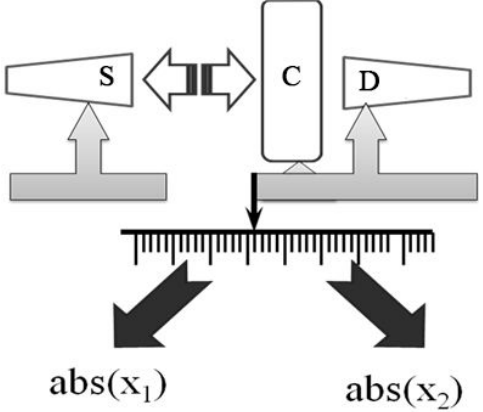

Fig. 1. Principal scheme of experimental setup. Two measurements were carried out for each sample and frequency.

\section{Acknowledgements}

The reported study was funded by RFBR according to the research project № 18-32-00328.

This work was carried out in the framework of state targets N 0035-2014-0206.

\section{References}

1. Vaks, V.L., Domracheva, E.G., Sobakinskaya, E.A., Chernyaeva, M.B. Exhaled breath analysis: physical methods, instruments, and medical diagnostics // Phys.-Usp. 2014. V. 57, No. 7. P. 684-701.

2. Vaks, V.L., Domracheva, E.G., Sobakinskaya, E.A., Chernyaeva, M.B. Methods and Instruments of High-Resolution Transient THz Spectroscopy for Diagnostics of Socially Important Diseases // Phys. Wave Phen. 2014. V. 22, No. 3. P. 177-184.

3. Zhang, X.-C. Terahertz wave imaging: horizons and hurdles // Phys. Med. Biol. 2002. V. 47. P. 3667-3677.

4. Alexandrov, B.S., Phipps, M.L., Alexandrov, L.B., Booshehri, L.G. et. al. Specificity and Heterogeneity of Terahertz Radiation Effect on Gene Expression in Mouse Mesenchymal Stem Cells // s. rep. 2013. V. 1184, No. 3. P. $1-8$.

5. Alexandrov, B.S., Gelev, V., Bishop, A.R., Ushevaet, A., et al. DNA Breathing Dynamics in the Presence of a Terahertz Field // Phys. Lett. A 2010. V. 374, No. 10. P.1214-1217.

6. Markelz, A., Whitmire, S., Hillebrecht, J. Birge, R. $\mathrm{THz}$ time domain spectroscopy of biomolecular conformational modes // Phys. Med. Biol. - 2002 - 47; URL: stacks.iop.org/PMB/47/3797V. 57, No. 7. P. 684-701.

7. Vol'kshtejn, M.V. Biofizika // Nauka, Moscow 1988

8. Koshland, D.E. Application of a Theory of Enzyme Specificity to Protein Synthesis // Proc. Natl. Acad. Sci. U.S.A. 1958. V.44, No.2. P.98-104.

9. Zuev, V.A. Priony - osobyj klass vozbuditelej medlennyh infekcij cheloveka i zhivotnyh // RMZh 2013. V. 30. P. $1559-1566$.

10. Vaks, V.L., Domracheva, E.G., Sobakinskaya, E.A., Chernyaeva, M.B. Exhaled breath analysis: physical methods, instruments, and medical diagnostics // Phys.-Usp. 2014. V. 57, No. 7. P. 684-701.

1. Vaks, V.L., Domracheva, E.G., Sobakinskaya, E.A., Chernyaeva, M.B. Exhaled breath analysis: physical methods, instruments, and medical diagnostics // Phys.-Usp. 2014. V. 57, No. 7. P. 684-701. 
Hastings, D. E., Wang, J. K. The radiation impedance of electrodynamic tether with end connectors // Geophys. Res. Lett. 1987. V. 14, No. 6. P. 519-522.

2. Bergman, D. J., Stroud, D. Physical properties of macroscopically inhomogeneous media // Solid State
Physics: Advances in Research and Applications, edited by H. Ehrenreich and D. Turnbull. New York: Academic Press. 1992. V. 46. P. 147-269. 